$\mathrm{CI}=1.5 \%-3.8 \%)$ and TSAs $(0.3 \%, 95 \% \mathrm{CI}=0.1 \%-0.8 \%)$ were shown in figure 1 (figure 1). Subgroup analysis indicated their higher prevalence among male $(22.0 \%$, 95\% CI $=16.4 \%$ $28.2 \%)$ than female $(14.0 \%, 95 \% \mathrm{CI}=8.2 \%-21.0 \%)$, and Caucasian $(25.9 \%, 95 \% \mathrm{CI}=16.4 \%-28.2 \%)$ than Asian $(14.6 \%$, $95 \% \mathrm{CI}=11.6 \%-18.2 \%)$. The prevalence of SSPs was also higher among Caucasian (2.9\%, 95\% CI=1.5\%-4.7\%) than Asian $(0.7 \%, 95 \% \mathrm{CI}=0.4 \%-1.2 \%)$. However, their prevalence were similar between male $(2.9 \%, 95 \% \mathrm{CI}=1.0 \%-5.7 \%)$ and female $(2.4 \%, 95 \% \mathrm{CI}=0.8 \%-4.9 \%)$.

Conclusions This was the first systematic review and metaanalysis to determine the overall prevalence of serrated lesions and their different subtypes. The pooled prevalence estimates can be used as indicators for establishing high-quality CRC screening programmes. Future studies should be performed to evaluate the factors independently associated with the presence of serrated lesions, which could inform clinical practice to enhance their detection rate.

\section{IDDF2019-ABS-0249 COMPARISON OF POST ENDOSCOPIC MUCOSAL RESECTION BLEEDING BETWEEN EPINEPHRINE AND HYPERTONIC SALINE SUBMUCOSAL INJECTION FOR COLON POLYPS IN PATIENTS TAKING ANTITHROMBOTIC AGENTS}

${ }^{1}$ Daisuke Yamaguchi* ${ }^{2}$ Yasuhisa Sakata, ${ }^{1}$ Yuki Takeuchi, ${ }^{1}$ Kei Ikeda, ${ }^{1}$ Wataru Yoshioka ${ }^{1}$ Amane Jyubashi, ${ }^{1}$ Tomohito Morisaki, ${ }^{1}$ Keisuke Ario, ${ }^{1}$ Seiji Tsunada. 'Ureshino Medical Center, Japan; 'Department of Internal Medicine and Gastrointestinal Endoscopy, Saga Medical School, Japan

\subsection{6/gutjnl-2019-IDDFabstracts.209}

Background The present study has investigated the comparison of the clinical outcomes of prophylactic submucosal saline-epinephrine injection and hypertonic saline injection for colon polyps in patients taking antithrombotic agents.

Methods We retrospectively investigated the medical records of 183 patients who taking antithrombotic agents among 891 patients who underwent colonic endoscopic mucosal resection (EMR) from January 2013 to March 2018. The patients were divided into two groups: group A, 92 patients with saline-epinephrine injection use; and group B, 91 patients with hypertonic saline $(10 \% \mathrm{NaCl})$ injection use. Clinical characteristics, type of antithrombotic agents, treatment outcomes and adverse events were compared between the two groups. (figure 1)

Results The mean age was 73.5 and 74.0 years in group A and $B$, sex and patient characteristics were not different between the two groups. The main antithrombotic agents were low-dose aspirin (A: 33, B: 31 patients), warfarin (A: 9,

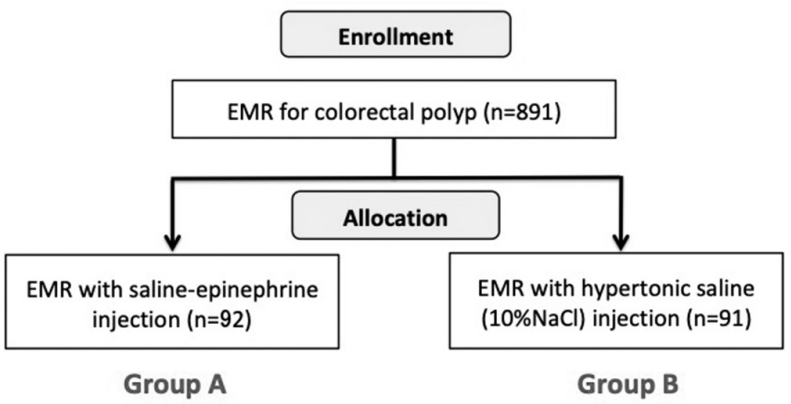

Abstract IDDF2019-ABS-0249 Figure 1 Flow diagram of the study
B: 22 patients), and clopidogrel (A: 20, B: 11 patients). Before colonic EMR, heparin bridge therapy (A: 20, B: 23 patients) and cilostazol replacement (A: 15, B: 11 patients) were equally performed.

There was no significant difference between the two groups in characteristics of colonic polyps. En bloc resection rate was similar successfully between the two groups $(94.6 \%$ vs 97.8\%). EMR procedure was performed by trainees more frequently in group A than B $(73.9 \%$ vs $48.4 \%$ : $\mathrm{p}<0.001)$. There were no significant differences in post EMR bleeding (7.6\% vs $4.4 \%)$, immediate EMR bleeding (6.5\% vs $3.3 \%)$ and other adverse events between the two groups.

Conclusions The post EMR bleeding in patients taking antithrombotic agents was similar between saline-epinephrine and hypertonic saline submucosal injection.

\section{IDDF2019-ABS-0252 EFFECT OF MULTIDONOR INTENSIVE FECAL MICROBIOTA TRANSPLANTATION BY CAPSULES FOR ACTIVE UNCREATIVE COLITIS: A PROSPECTIVE TRIAL}

\author{
${ }^{1}$ Yanyun Fan*, 'Qiongyun Chen, ${ }^{2}$ Bangzhou Zhang, ${ }^{2}$ Zhangran Chen, ${ }^{1}$ Qingwen Huang, \\ ${ }^{1}$ Hongzhi Xu, ${ }^{1}$ Jianlin Ren, ${ }^{2}$ Xiang Zhang. ${ }^{1}$ Zhongshan Hospital Department of \\ Gastroenterology, Xiamen University, China; ${ }^{2}$ Institute for Microbial Ecology, Medical \\ College of Xiamen University, China
}

\subsection{6/gutjnl-2019-IDDFabstracts.210}

Background Fecal microbiota transplantation (FMT) can induce remission in patients with ulcerative colitis(UC). We aim to establish the efficacy of multidonor, easy way FMT by capsules in active UC, and identify the factors associated with response to therapy.

Methods We conducted a prospective trial in Zhongshan Hospital Affiliated to Xiamen University, Xiamen, China. A total of 28 adults with active UC (Mayo score 4-11) were enrolled in this trial, followed by capsules 3 days one week. One donor's fecal microbiota was made into one capsule, and fecal microbiota transplantation was each derived from two or three unrelated donors. The primary outcome was steroid-free clinical remission with endoscopic remission or response. We performed $16 \mathrm{~S}$ rDNA stool analysis to access associated microbial changes. Based on LC-MS/MS technology metabonomic researches were carried out to study metablome of the serum. Results The primary outcome with clinical remission was achieved in 16 out of 28 patients $(57.14 \%)$, and 11 out of 22 patients $(50 \%)$ had the musical healing. No adverse events were identified in these patients. The effects have no significant relationship with the age, gender, severity and extent of disease.FMT increased microbial diversity and altered composition. After FMT, unlike non-responders, the intestinal flora structure of responders was close to the donor, and the significant change began 1 week after transplantation. After FMT, responders had enrichment of Alloprevotella compared with non-responders and had increased levels of taurochenodeoxycholate and taurocholate. Escherichia-Shigella were decreased after FMT in responders, but no change in non-responders. The correlation analysis between the differential metabolites and the differential flora showed that the bile acid-related metabolites were positively correlated with the abundance of Alloprevotella and Prevotella_9.

Conclusions Intensive-dosing, multidonor, FMT by capsules induces clinical remission in active ulcerative colitis and is associated with the changes of intestinal flora. Alloprecotella 